\title{
Heat Transfer Enhancement in a Lithium-ion Cell Through Improved Material-Level Thermal Transport
}

\section{Vivek Vishwakarma ${ }^{1}$, Chirag Waghela ${ }^{1}$, Zi Wei ${ }^{2}$, Ravi Prasher ${ }^{3}$, Shrikant C Nagpure ${ }^{4}$, Jianlin $\mathrm{Li}^{4}$, Fuqiang Liu ${ }^{2}$, Claus Daniel ${ }^{4,5}$, and Ankur Jain ${ }^{1, *}$}

1 - Department of Mechanical \& Aerospace Engineering, The University of Texas at Arlington, Arlington, TX, USA 75019

2 - Department of Materials Science and Engineering, The University of Texas at Arlington, Arlington, TX, USA 75019

3 - Energy Storage and Distributed Resources Division, Lawrence Berkeley National Laboratory, Berkeley, CA, USA 94720

4 - Energy and Transportation Science Division, Oak Ridge National Laboratory, Oak Ridge, TN, USA 378316472 ,

5 - Bredesen Center for Interdisciplinary Research and Graduate Education, The University of Tennessee, Knoxville TN 37996

* Corresponding Author: 500 W First St, Rm 211, Arlington, TX 76019, USA, email: jaina@uta.edu; Ph.: +1-817-272-9338, Fax: +1-817-272-2952. 


\begin{abstract}
While Li-ion cells offer excellent electrochemical performance for several applications including electric vehicles, they also exhibit poor thermal transport characteristics, resulting in reduced performance, overheating and thermal runaway. Inadequate heat removal from Li-ion cells originates from poor thermal conductance within the cell. This work identifies the rate-limiting material-level process that dominates overall thermal conduction in a Li-ion cell. Results indicate that thermal characteristics of a Li-ion cell are largely dominated by heat transfer across the cathode-separator interface rather than heat transfer through the materials themselves. This interfacial thermal resistance contributes around $88 \%$ of total thermal resistance in the cell. Measured value of interfacial resistance is close to that obtained from theoretical models that account for weak adhesion and large acoustic mismatch between cathode and separator. Further, to address this problem, we enhance the adhesion of this interface using amine-based chemical bridging. This results in four-times lower interfacial thermal resistance without deterioration in electrochemical performance, thereby increasing effective thermal conductivity by three-fold. This improvement is expected to reduce peak temperature rise during operation by $60 \%$. By identifying and addressing the material-level root cause of poor thermal transport in Li-ion cells, this work may contribute towards improved thermal performance of Li-ion cells.
\end{abstract}

Keywords: Li-ion Cell; Thermal Management; Heat Transfer; Interfacial Thermal Conductance; Thermal Runaway. 


\section{Introduction}

Inadequate heat removal in Li-ion battery cells [1-3] is a key technological challenge needed to be overcome for widespread adoption of electric vehicles (EVs) [4-5]. Despite excellent energy storage and conversion characteristics [1-3] Li-ion cells continue to suffer from poor heat dissipation. Inefficient heat dissipation and overheating in a Li-ion cell directly affects performance and reliability, in addition to presenting significant safety concerns related to thermal runaway [3-4]. A full charging of an EV battery pack within a few minutes is likely to generate significant amount of heat [6], which may be a limiting factor in the goal of fast charging of EVs. Even though the heat generation rate in a Li-ion cell is not particularly high (for example, around 4W for a 26650 cell undergoing 6C discharge [6]), its poor thermal conductivity [7] results in large temperature rise and thermal gradients within the cell [8-9], leading, in extreme cases, to a thermal runaway situation $[3,10]$. In order to maintain the cell temperature within safe limits, performance is often sacrificed by operating the cell less aggressively than it could be, overdesigning the battery pack and providing an elaborate external cooling system [5,11]. Each of these measures results in reduced performance and reliability, and does not address the root cause of inefficient thermal dissipation.

Despite the importance of thermal transport in Li-ion cells, a fundamental understanding of underlying mechanisms is lacking. While materials in a Li-ion cell are reasonably welloptimized for electrochemical performance [12-14], relatively lesser research has been carried out to measure, understand and optimize thermal transport within the Li-ion cell materials. Our recent work showed that thermal conduction within the cell is the dominant mechanism in determining the overall thermal performance of the cell, as opposed to heat transfer from outer surface of the cell [8-9]. Our recent measurements on Li-ion cells indicated strong anisotropy in 
thermal conduction, and a poor thermal conductivity of $0.15-0.20 \mathrm{~W} / \mathrm{mK}$ in the direction normal to the electrodes [7]. This value is as poor as that of typical polymers [15]. It is clearly important to understand and improve the material-level origin of this poor thermal conductivity. Thermal conductivity of typical electrode and separator materials measured separately is around 2-5 W/m$\mathrm{K}$ [16-18] and 0.3-1.0 W/m-K [19-21] respectively. Other components such as current collectors (copper or aluminum) and anode (graphite) have even higher thermal conductivity. Using a simple series resistance model that accounts for only material thermal conductivities, the effective thermal conductivity of the thermal unit cell in the direction normal to electrode surface is found to be around $1.76 \mathrm{~W} / \mathrm{mK}$, about 8 times higher than the experimentally measured celllevel thermal conductivity of a 18650 and 26650 Li-ion cells [7]. This suggests that thermal transport in a Li-ion cell may be dominated by interface thermal resistance between materials rather than thermal resistance of the materials themselves. This may be occurring in a Li-ion cell because separator and electrodes are merely pressed on to each other during cell manufacturing, which is likely to result in poor surface adhesion and hence large thermal contact resistance [22]. Dominance of thermal conduction processes by interfacial thermal resistance is encountered in other microsystems as well, including microelectronics and microelectromechanical systems (MEMS) [23-25].

Figure 1A shows a cut-out of a typical Li-ion cell, indicating the spirally wound stack of electrodes, separator and current collectors. While in-plane heat flow can occur along high thermal conductivity materials, such as current collectors, heat flow in the out-of-plane direction of the stack must conduct through each of these materials as well as through their interfaces before it can be convected away from the outer surface of the cell. The thermal unit cell that repeats itself in the direction normal to the electrode surface is shown in Figure 1B. The unit cell 
comprises multiple layers of anode, cathode, separator and current collectors, and offers various material and interfacial thermal resistances associated with these materials. Note that the anode/cathode materials are coated on both sides of the current collectors. In order to improve cell-level thermal performance, it is critical to measure the thermal resistances in this unit cell, and identify and improve the dominant, rate-limiting steps in the thermal conduction process.

In this paper, we show that thermal conduction within this thermal unit cell is dominated by a large thermal contact resistance (TCR) at the separator-cathode interface, contributing around $88 \%$ of total thermal resistance and temperature rise. On the other hand, TCR at the anode-separator interface is negligible. Our measured TCR correctly predicts the recently reported [7] poor cell-level thermal conductivity of Li-ion cells despite the large thermal conductivities of constituent materials. We show that the large TCR is caused by weak adhesion between the separator and cathode materials. Dominance of the TCR shows that improving thermal conductivity of electrode or separator is unlikely to result in significant enhancement in overall thermal performance. We demonstrate $4 \mathrm{X}$ improvement in thermal contact resistance by chemically bridging the interface with amine chemistry [23, 26-27] without affecting electrochemical performance of the cell. This improvement is expected to result in more than $3 \mathrm{X}$ improvement in cell-level thermal conductivity and $60 \%$ reduction in peak temperature rise during cell operation at 7C discharge rate. By identifying and alleviating the rate-limiting process in thermal conduction in a Li-ion cell, this work provides the technological basis for significantly reducing the overheating problem in Li-ion cells, resulting in improved performance, safety and reliability.

\section{Experimental Methods}

\subsection{Electrode and separator sample preparation}


Positive electrode $\left(\mathrm{LiCoO}_{2}\right)$ and separator are extracted from 18650 commercial Li-ion batteries. The cell is first discharged completely. The metal casing enclosing the spirally-wound electrodes and separator is opened in a laminar flow fume hood. Separator and positive electrode are peeled off carefully from the separator-electrode stack. All experiments are carried out on these materials extracted from a 18650 cell, and not on the 18650 cell itself.

\subsection{Thermal measurements}

Total thermal resistance across a material stack is determined by sandwiching the stack between two identical copper blocks [28]. Figure 2 shows a schematic of the experimental setup. A picture is also shown in the inset in Figure 3. The faces of the copper blocks are polished, first with a 120 grit sandpaper belt on a LECO BG-30 polisher, and then with a 1200 grit sandpaper embedded with $0.05 \mu \mathrm{m}$ alumina microparticles on LECO Vp-150 polisher. In one of the blocks, heat is generated using a thin Kapton heater affixed on one of the faces. Heat is removed from the other block using cooling water from a chiller passing through a $1 \mathrm{~mm}$ diameter through-hole drilled close to one of the faces. Temperature measurement is carried out using T-type thermocouples inserted in seven holes of $1.0 \mathrm{~mm}$ diameter in each block. The holes are spaced closer to each other near the surfaces contacting the material stack for greater accuracy near the stack. Holes are drilled in the horizontal plane, which minimizes heat loss down the thermocouple wires. Omega CC High Temperature cement is used to ensure good thermal contact between thermocouple tips and the copper block. The copper blocks are then insulated on all faces except the ones contacting the material stack to minimize stray heat losses. Kapton heater attached to the top block is electrically heated using a power source. Since the electrical resistance of the heater does not vary appreciably in the temperature range of interest, it serves as a source of constant heat flux throughout the experiment. A Keithley 2100 digital multimeter is 
used to monitor voltage across the heater. Thermocouple temperature measurements are recorded using a National Instruments 9213 DAQ and LabView software with $1 \mathrm{~Hz}$ frequency. The experiment continues until steady state is reached. Steady-state temperature measurements from embedded thermocouples are extrapolated to determine the temperature drop across the sample. Heat flux passing through the blocks is determined from the slope of the temperature curve in the copper blocks. Total thermal resistance of the material stack is determined from the ratio of temperature drop and heat flux.

\subsection{Electrochemical testing}

A split flat cell with active area of $2.54 \mathrm{~cm}^{2}$ from MTI Corp. is employed for electrochemical testing. The baseline and modified cathodes, together with commercial Celgard 2500 separators and lithium anodes extracted from a 18650 cell as described in Section 2.1 are

used as cell components. During assembly of the cell in a controlled atmosphere glove box, additional home-made 1.0 $\mathrm{M} \mathrm{LiPF}_{6}$ in mixed solvents of ethylene carbonate (EC)-dimethyl carbonate (DMC) (1:1 by volume) is added to compensate for electrolyte loss. After being kept at rest for 24 hours, the cell is first charged at $1.5 \mathrm{~mA}$ (roughly corresponding to a rate of $0.33 \mathrm{C}$ ) until the voltage reached $4.0 \mathrm{~V}$, then was held at a constant voltage of $4.0 \mathrm{~V}$ for 30 minutes, followed by a second period of rest for 20 minutes. After that, the cell is discharged at $1.5 \mathrm{~mA}$ until the voltage dropped below 2.5 V. EIS experiments are conducted over a frequency range from $0.1 \mathrm{mHz}$ to $100 \mathrm{MHz}$ at open circuit with an amplitude of $10 \mathrm{mV}$ while the cells are fully discharged. Both battery performance and EIS tests are performed on a Parstat 2273 potentiostat/galvanostat.

\subsection{Surface Modification}


In order to enhance interfacial thermal conductance, surface modification is carried out on both cathode and separator surfaces. The separator is subjected to oxygen plasma in MicroRIE Series 800 Plasma System for 5-10 min at 30mTorr. Surface modification of cathode is carried out using amine chemistry [27]. Extracted cathode sample, after wash and dry is immersed into a $2 \% \mathrm{v} / \mathrm{v}$ solution of (3-Aminopropyl)triethoxysilane in ethanol, and left for incubation for 30 minutes in room temperature. This forms an amine self-assembled amine monolayer (SAM).

\section{Theoretical Modeling for Electrode-Separator TCR}

Multiple features of the cathode-separator interface must be accounted for in determining the interfacial thermal contact resistance: a) Large acoustic mismatch between cathode and separator, b) Weak adhesion between cathode and separator, c) Large porosity of separator resulting in reduction in effective area of contact, and, d) Further reduction in area of contact [29], due to the fibrous nature of the separator ( 1 $\mu \mathrm{m}$ fiber diameter) [30]. The baseline

contact conductance per unit area (g) between two material surfaces is given by [31]

$$
g=\Gamma \cdot \frac{c \cdot v}{2}
$$

where $\Gamma$ is the integrated phonon transmissivity, $c$ the specific heat for polyethylene and $v$ is the speed of phonons in polyethylene. Since the phonon speed is smaller in polyethylene, conductance must be calculated from the polyethyelene side, wherein $\Gamma$ is integrated till the critical angle. The conductance shown in equation (1) is valid only for interface between two flat substrates. This conductance is scaled to account for porosity and non-flat nature of the substrate. The effective thermal contact conductance is thus given by 


$$
g=\frac{a}{R} \cdot \phi \cdot \Gamma \cdot \frac{c \cdot v}{2}
$$

where $\phi$ is the porosity, $a$ is the contact width and R is the radius of the fiber. $a$ is computed using the well-known Johnson-Kendall-Roberts adhesion mechanics model [32] for cylinders [33]. $R$ is assumed to be $1 \mu \mathrm{m}$ in these calculations (typical radius of fibers in the separator [34]). Porosity is assumed to be $50 \%$.

Note that critical angle of refraction for $\mathrm{LiCoO}_{2}$ and Polyethylene interface is very low due to large mismatch in phonon speed, which is expected to result in very high interface resistance. On the other hand, the critical angle between graphite (anode) and polyethylene is much larger as the phonon speeds are much closer. Therefore, the contact conductance is expected to be much higher for this interface compared to $\mathrm{LiCoO}_{2} /$ Polyethylene interface.

\section{Results and Discussion}

\subsection{TCR Measurement}

The thermal unit cell shown in Figure 1B comprises of two half-cells, one each on the anode and cathode sides. The material thermal resistances in the two half-cells are expected to be similar to each other, due to the relatively similar values of thermal conductivity of anode and cathode materials [16-18, 35]. However, theoretical modeling results discussed in section 4.2 show that thermal contact resistance between anode and separator, $R_{\text {sep-anode }}$ is two orders of magnitude lower than thermal contact resistance between cathode and separator, $R_{\text {sep-cathode }}$. As a result, the overall thermal conductivity of the entire unit cell is expected to be dominated by the thermal resistance of the cathode-side half-cell. Consequently, we focus on measurement and reduction of total thermal resistance of the cathode half-cell, shown in Figure 1B. The total thermal resistance of the cathode half-cell is given by 


$$
R_{\text {total }}=\frac{L_{\text {sep }}}{k_{\text {sep }}}+2 \cdot R_{\text {sep-cathode }}+\frac{L_{\text {cathode }}}{k_{\text {cathode }}}+\frac{L_{c c}}{k_{c c}}+2 \cdot R_{\text {cathode-cc }}
$$

where subscripts sep and $c c$ refer to the separator and current collector respectively, $L$ and $k$ are thickness and thermal conductivity respectively. $R_{\text {sep-cathode }}$ and $R_{\text {cathode-cc }}$ refer to the thermal contact resistances at the separator-cathode and cathode-current collector interfaces respectively. Note that $R_{\text {sep-cathode }}$ must be considered twice due to two cathode-separator interfaces in the unit cell.

We have neglected the contribution of the electrolyte to thermal transport. To verify this assumption, we carried out cell-level measurements on commercial $18650 \mathrm{Li}$-ion cells [7], which included electrolyte inside the cell. Our cell level thermal conductivity with electrolyte present matched with thermal conductivity determined from material-level measurements reported here.

Within the various thermal resistances shown in equation (3), $L_{c c} k_{c c}$ is expected to be very small (estimated $0.05-0.1 \mu \mathrm{Km}^{2} / \mathrm{W}$ ) due to the large thermal conductivity of current collector - typically made of copper or aluminum. Further, since the cathode material is coated directly on to the current collector foil, therefore, the two are in intimate thermal contact, with negligible thermal contact resistance compared to the interface between separator and cathode, which are merely pressed on to each other during cell manufacturing. As a result, the three dominant thermal resistances in this half-cell are expected to be the material resistance through cathode, material resistance through separator, and thermal contact resistance between cathode and separator (Figure 1C).

We carry out a series of measurements based on one-dimensional, steady-state, out-ofplane thermal conduction to determine these thermal resistances. In experiment 1 , a separatorelectrode stack is sandwiched between two copper blocks (Figure 2A). One of the blocks is 
heated using a Kapton heater, and the other is cooled using water flow, resulting in the setting up of linear one-dimensional heat flow through the sandwiched material. Figure 3 shows the steadystate temperature distribution measured using thermocouples for Experiment 1. Supplementary Figure S1 shows temperature measurement from one of the thermocouples as a function of time. Only steady-state measurements are used here, since at steady-state, all of the heat passing through the top copper block conducts across the sample. Total thermal resistance, $R_{\text {total }}$ through the separator-electrode stack is determined by extrapolating these temperature measurements and dividing by the heat flux flowing through the stack. In this case, the total thermal resistance is given by

$$
R_{\text {total }}=R_{\text {Cu-sep }}+\frac{L_{\text {sep }}}{k_{\text {sep }}}+R_{\text {Cu-cathode }}+\frac{L_{\text {cathode }}}{k_{\text {cathode }}}+R_{\text {sep-cathode }}
$$

where, as noted prior, thermal resistance through the current collector and the cathode-current collector interface is negligibly small.

Thermal conductivities of cathode and separator materials have been measured in the past [16-19]. Based on a value of $0.5 \mathrm{~W} / \mathrm{mK}$ for the separator thermal conductivity reported by two of the co-authors [19], the material thermal resistance for the separator is found to be $L_{\text {sep }} / k_{\text {sep }}=50$ $\mu \mathrm{Km}^{2} / \mathrm{W}$. Based on thermal measurements on various cathode materials [16-18], the cathode thermal conductivity is assumed to be $5.4 \mathrm{~W} / \mathrm{mK}$. As a result, the material thermal resistance of the cathode is around $11 \mu \mathrm{Km}^{2} / \mathrm{W}$, which is even lower than the thermal resistance of the separator.

In contrast to material thermal resistances, the thermal contact resistances appearing in equation (4) are largely unknown. While $R_{\text {Cu-sep }}$ and $R_{\text {Cu-cathode }}$ occur due to the experimental 
setup, $R_{\text {sep-cathode }}$ is intrinsic to the Li-ion material stack. We carried out two sets of experiments, shown in Figures 2B and 2C to determine these thermal contact resistances.

In order to determine, $R_{\text {Cu-sep }}$, the contact resistance between copper block and separator, the total thermal resistance across a single separator sample sandwiched between copper blocks is measured (Experiment 2, shown in Figure 2B). Since there are two interfaces between copper and separator, the total resistance in this case is given by

$$
R_{\text {total }}=2 R_{\text {Cu-sep }}+\frac{L_{\text {sep }}}{k_{\text {sep }}}
$$

Measurement of $R_{\text {total }}$, together with the material thermal resistance of the separator based on prior measurements, results in $R_{\text {Cu-sep }}=625 \mu \mathrm{Km}^{2} / \mathrm{W}$ from equation (5).

In Experiment 3, a cathode sample coated on both sides of a current collector is sandwiched between the copper blocks (Figure 2C). In this case, the total thermal resistance is given by

$$
R_{\text {total }}=2 \cdot R_{\text {Cu-cathode }}+\frac{L_{\text {cathode }}}{k_{\text {cathode }}}
$$

where, similar to Experiment 1, we neglect the thermal resistance due to the current collector and the thermal contact resistance between cathode and current collector.

Using recently reported values for thermal conductivity of the cathode [16-18] together with measurement of $R_{\text {total }}$ for Experiment 3, we determine the thermal contact resistance $R_{C u \text { - }}$ cathode to be $345 \mu \mathrm{Km}^{2} / \mathrm{W}$.

Experiments 2 and 3 provide two of the thermal contact resistances appearing in equation (4), leaving only the thermal contact resistance between separator and cathode as an unknown. Thus, a measurement of the total thermal resistance of the separator-cathode stack in Experiment 
1 can be used to determine $R_{\text {sep-cathode }}$. This value is found to be $420 \mu \mathrm{Km}^{2} / \mathrm{W}$, much larger than the material resistances due to separator and cathode.

These experiments provide measurements of all thermal resistances involved in the cathode-side half-cell shown in Figure 1B. Left side of Figure 4 summarizes the contributions of these thermal resistance terms to total thermal resistance. Note that the interface thermal resistance $R_{\text {sep-cathode }}$ must be accounted for twice since there are two separator-cathode interfaces in the unit cell. In addition, the unit cell also comprises two separator layers. Our measurements indicate that thermal contact resistance between separator and cathode dominates the thermal conduction process, accounting for around $88 \%$ of the total thermal resistance of the unit cell. Consequently, material-level optimization of thermal transport in a Li-ion cell must focus on this rate-limiting process by improving thermal contact between these two materials. Merely improving material thermal conductivities of separator and/or electrodes without addressing conduction through the interface [37] is unlikely to result in significantly improved overall thermal performance. For example, doubling the separator or cathode thermal conductivity results in only $4.4 \%$ and $0.5 \%$ reduction in total thermal resistance respectively. It is imperative, instead, to improve the thermal contact resistance between cathode and separator.

\subsection{Theoretical Modeling Results}

Material properties used for the calculation of thermal contact resistance are listed in Table 1. The baseline thermal contact resistance calculated using Acoustic Mismatch Model (AMM) [36] based on properties of the cathode and separator is $1.1 \mu \mathrm{Km}^{2} / \mathrm{W}$. We account for the other effects listed in section 3 through the following analysis: we assume that the separator is in contact with the cathode through weak van der Waals adhesion due to the two being merely 
pressed against each other during the Li-ion cell manufacturing process. One of the co-authors has shown [31] that weak adhesion leads to dramatic reduction in transmissivity of phonons at the interface leading to high interface resistance. For van der Waals adhesion, the adhesion energy is around $50 \mathrm{~mJ} / \mathrm{m}^{2}$ [34]. The transmissivity of phonons for this adhesion energy is 10 to 20 times lower than that of AMM [32] computed for a strongly bonded interface. Accounting for this effect results in thermal contact resistance in the range of $11-22 \mu \mathrm{Km}^{2} / \mathrm{W}$. Furthermore, assuming 50\% porous separator [12], the expected thermal contact resistance increases to 22-44 $\mu \mathrm{Km}^{2} / \mathrm{W}$. Finally, since the separator is made of polymer fibers only the deformed part of the fiber due to adhesive forces participates in heat transfer [29]. This deformed part effectively creates a nano-constriction through which heat must conduct. The area of the deformed part is calculated using Johnson-Kendall-Roberts theory [32] for cylinders [33]. Based on the properties of polypropylene, the ratio of width of this constriction and radius of the fiber is 0.084 , i.e. the effective contact resistance increases by a factor of $1 / 0.084=11.85$. Using this number, the thermal contact resistance between cathode and separator is found to be in the range of $260-520 \mu \mathrm{Km}^{2} / \mathrm{W}$ which is very close to the experimental measurements discussed in section 4.1. On the other hand, due to much larger critical angle between anode and separator compared to the cathode-separator interface, the thermal contact resistance between anode and separator is found to be in the range of $2-4 \mu \mathrm{Km}^{2} / \mathrm{W}$, which represents around two orders of magnitude greater thermal contact conductance than the cathode-separator interface. This shows that the anode-separator interface plays an insignificant role in determining overall thermal performance of the unit cell.

\subsection{TCR Enhancement}


In order to improve thermal dissipation in a Li-ion cell by reducing the dominant thermal resistance, we carry out experiments to investigate improved thermal adhesion between the separator and cathode through surface treatment. This is motivated by past work by one of the co-authors [23] in which improved adhesion between surfaces using chemical surface modification led to significant decrease in thermal contact resistance [23]. It is well known that thermal contact resistance is correlated with surface adhesion energy [22-23], which is the lowest for van der Waals based adhesion, and can be improved through chemically bridging the interface. Three experiments are carried out as illustrated in Figure 5. Our experiments are based on a self-assembled monolayer of amine groups on the cathode surface, and plasma treatment of the separator. Amine groups are commonly used for functionalizing glass surfaces with DNA and other biomolecules [26-27]. Plasma treatment of the separator was motivated by well-known enhancement of surface adhesion of polymers and other materials caused by plasma treatment [38-39]. Four experiments that combine surface modification with plasma treatment, as shown schematically in Figure 5 are carried out. Experiment A is the baseline case. In Experiments B and C, surface modification of cathode and plasma treatment of separator are carried out, respectively. In Experiment D, both are carried out. In each case, we measure the resulting thermal contact resistance between the separator and cathode using the technique described in section 2.2. Figure 6 plots the steady-state temperature measurements from each thermocouple in the experimental setup for Experiments A-D. For each case, the temperature drop across the separator-electrode sample, and hence total thermal resistance is obtained by extrapolation of data shown in Figure 6. Starting from the imposition of Joule heating on the experimental setup, temperature drop across the sample increases with time, and eventually reaches a steady-state, as shown in Supplementary Figure S2. Only the steady-state temperature difference is considered, 
since heat is being stored in the copper blocks prior to steady state, due to which the temperature difference across the sample does not accurately reflect its thermal resistance. Data presented in Figure 6 shows significant reduction in total thermal contact resistance due to surface modification. When the material resistances and contact resistances with the copper blocks are subtracted out, similar to previous section, $R_{\text {sep-cathode }}$ was found to reduce by $26 \%, 41 \%$ and $78 \%$ compared to baseline measurements for experiments B, C and D respectively. The largest reduction is found when both separator and cathode surfaces are treated (Experiment D), in which case, the measured TCR of $90 \mu \mathrm{Km}^{2} / \mathrm{W}$ is $4 \mathrm{X}$ lower than the baseline case, as shown on the right side of Figure 4 . The total thermal resistance of the unit cell reduces from $951 \mu \mathrm{Km}^{2} / \mathrm{W}$ to $291 \mu \mathrm{Km}^{2} / \mathrm{W}$. This extent of TCR reduction is consistent with $4 \mathrm{X}$ and $6 \mathrm{X}$ reduction reported by O’Brien, et al. [22] and Kaur, et al. [23] respectively, using an organic bonding layer across $\mathrm{Cu} / \mathrm{SiO}_{2}$ interface, and amine-terminated carbon nanotubes respectively.

By reducing the thermal resistance responsible for the largest contribution to overall thermal conduction in the Li-ion cell, the surface modification results in improved overall thermal conductance of the thermal unit cell, as summarized on the right side of Figure 4.

In order to investigate the effect of surface modification of the cathode on battery performance, electrochemical tests are performed in a split flat cell. Figure 7A shows the complete charge-rest-discharge curves for the baseline (Experiment A) and modified (Experiment D) electrodes. The charging potential is about $32 \mathrm{mV}$ lower than the baseline, and the discharge potential is about $37 \mathrm{mV}$ higher than the baseline, indicating more available energy and higher round trip energy efficiency. This may be due to lower interfacial impedance. The voltage efficiency, evaluated by the ratio of plateau potentials during discharge and charge, is improved by around $2 \%$. To determine the cell internal resistance, electrochemical impedance 
spectroscopy (EIS) is conducted at fully discharged condition. Figure 7B shows that the internal resistance of the cell with modified cathode is reduced by about 3 Ohms, while the charge transfer resistance (diameter of the high-frequency semi-circle) does not change significantly. This indicates that the improved cell performance and reduced internal resistance may be due to a enhanced surface contact between the modified cathode and separator.

Further work must be carried out to fully optimize the specific nature of the chemical bridge for both thermal and electrochemical performance. Long-term stability of the chemical bridging in an electrochemically active environment needs to be further analyzed by evaluating electrochemical stability of the chemical species in the full electrochemical potential window of operation, and potential side reactions with chemical species present in the cell.

\subsection{Influence on cell-level thermal performance}

Our material-level measurements are consistent with cell-level thermal conductivity measurements [7]. The thermal conductivity of the thermal unit cell, accounting for the measured value of the thermal contact resistance between cathode and separator, is found to be 0.24 $\mathrm{W} / \mathrm{mK}$, which is very close to cell-level measurements of $0.15-0.20 \mathrm{~W} / \mathrm{mK}$ for 26650 and 18650 Li-ion cells [7]. If the thermal contact resistance measured here is not accounted for, and only material thermal resistances are considered, then the effective cell-level thermal conductivity is found to be $1.76 \mathrm{~W} / \mathrm{mK}$, which is much higher than cell-level measurements [7].

Figure 8 plots the expected overall cell-level thermal conductivity as a function of thermal contact resistance between cathode and separator. Baseline (Experiment A) and improved (Experiment D) values of the thermal contact resistance are also shown on the $\mathrm{x}$-axis. Figure 8 indicates an improvement in overall thermal conductivity from $0.24 \mathrm{~W} / \mathrm{mK}$ (baseline) to $0.76 \mathrm{~W} / \mathrm{mK}$ (experiment D). There is potential for even steeper improvement in overall thermal 
conductivity by reducing TCR more through further optimization of the separator-electrode interface. If the TCR can be eliminated completely, the cell-level thermal conductivity can be improved up to $1.76 \mathrm{~W} / \mathrm{mK}$.

Figure 8 also plots the peak temperature rise in a $26650 \mathrm{Li}$-ion cell operating at 7C discharge rate in ambient conditions as a function of TCR. The peak temperature rise is computed using our recently reported cell-level thermal model that solves the governing energy equations to predict the temperature distribution in a Li-ion cell as a function of its thermophysical properties [8]. Figure 8 shows a significant reduction in peak temperature rise due to TCR reduction and the consequent improvement in thermal conduction within the cell. An additional benefit of TCR reduction demonstrated here is a reduction in temperature gradients within the cell, which is very desirable for balanced and safe cell operation [3]. Temperature gradient within the cell is expected to reduce from $23^{\circ} \mathrm{C}$ to $9.5^{\circ} \mathrm{C}$ between the baseline TCR and enhanced TCR - a reduction of over $58 \%$. This is made possible by more effective heat removal from the core of the cell, which is a significant challenge at present due to poor cell thermal conductivity. Reductions in both peak temperature and temperature gradient are expected to result in reduced risk of failure, improved reliability and enhanced performance [3]. For example, the thermal head made available by the reduced cell temperature could be made use of by allowing the cell to operate at a higher discharge rate than present, thereby improving performance. This may also reduce the number of cells needed in a battery pack for a certain power output, thereby reducing cost and weight.

Our material-level approach addresses the thermal management problem in Li-ion cells at its fundamental root cause, as opposed to other external approaches, such as cold plate cooling, external liquid cooling, embedded phase change cooling, etc. [3,11]. External cooling approaches 
are likely to have very limited benefits if the inherent cause of the poor thermal conduction within the cell is not addressed. In addition, our work shows the dominance thermal contact resistance over material resistances within the Li-ion material stack.

The key results presented here - the dominance of TCR as well as reduction in TCR due to surface modification - are both largely insensitive to the thermal conductivities of electrode and separator. Figure S3 in Supplementary Information shows plots of measured baseline and enhanced thermal contact resistances (experiments A and D) over a range of $k_{\text {sep }}$ and $k_{\text {elec }}$ respectively. Figure S3 shows that results from our measurements do not change significantly with changes in the material thermal conductivities within the range of values reported in the recent past [16-19].

Our results also indicate key trade-offs in thermal and electrochemical performance of a Liion cell. Much effort has focused on increasing the porosity and pore size in separators to facilitate ionic transport through the separator [12]. Our results indicate that doing so may actually be detrimental to thermal performance, since the rate-limiting thermal transport at the separator-electrode interface depends critically on good thermal contact between the two.

\section{Conclusions}

This work identifies interfacial thermal conduction between cathode and separator as the rate-limiting material-level component of heat transfer within a Li-ion cell, contributing around $88 \%$ of overall thermal resistance of the cell. Results also indicate dramatic enhancement of this interfacial thermal transport without affecting electrochemical resistance based on surface modification. Experimental measurements of the interfacial thermal contact resistance are in good agreement with predictions based on the acoustic mismatch model. Cell-level thermal property values based on these measurements are close to recent measurements on 18650 and 
26650 cells. Results indicate the possibility of significant improvement in cell-level thermal conductivity and reduction in operating temperature rise as a result of surface modification.

Future research should further investigate the effect of material properties on the separatorcathode contact, for example by changing the size and nature of separator polymer strands, etc. Various possible schemes for chemically bridging the interface must be evaluated experimentally and theoretically, for optimizing thermal and electrochemical effects, as well as for longer-term stability. Due to the closely coupled, multiphysics nature of a Li-ion cell, thermal transport must be evaluated in the context of the fundamental electrochemical processes within the cell. For inclusion in commercially manufactured batteries, integration of the surface modification process in the manufacturing flow must be considered and optimized.

\section{Acknowledgments}

A.J. and V.V. would like to acknowledge support from Provost's Office and Department of Mechanical and Aerospace Engineering, The University of Texas at Arlington. F.L. would like to acknowledge support from National Science Foundation (ECCS-1125588). Research at Oak Ridge National Laboratory, managed by UT Battelle, LLC, for the U.S. Department of Energy (DOE) under contract DE-AC05-00OR22725, utilized the DOE Battery Manufacturing R\&D Facility at ORNL sponsored by the Office of Energy Efficiency and Renewable Energy's Vehicle Technologies Office.

\section{References}

[1] J. Goodenough, K.-S. Park, J. Am. Chem. Soc. 135 (2013) 1167-1176.

[2] B. Scrosati, J. Garche, J. Power Sources 9 (2010) 2419-2430

[3] T.M. Bandhauer, S. Garimella, T. Fuller, J. Electrochem. Soc. 158 (2011) R1-R25.

[4] G. Karimi, X. Li, Int. J. Energy Res. 37 (2013) 13-24. 
[5] A.A. Pesaran, In: Proc. Adv. Automotive Battery Conf. (2001) Las Vegas, NV, USA.

[6] S.J. Drake, M. Martin, D.A. Wetz, J.K. Ostanek, S.P. Miller, J.M. Heinzel, A. Jain, J. Power Sources 285 (2015) 266-273.

[7] S.J. Drake, D.A. Wetz, J.K. Ostanek, S.P. Miller, J.M. Heinzel, A. Jain, J. Power Sources 252 (2014) 298-304.

[8] K. Shah, S.J. Drake, D.A. Wetz, J.K. Ostanek, S.P. Miller, J.M. Heinzel, A. Jain, J. Power Sources 258 (2014) 374-381.

[9] K. Shah, S.J. Drake, D.A. Wetz, J.K. Ostanek, S.P. Miller, J.M. Heinzel, A. Jain, J. Power Sources 271 (2014) 262-268.

[10] D. Lisbona, T. Snee, Process Safety \& Env. Protection 89 (2011) 434-442.

[11] M. Zolot, A. Pesaran, M. Mihalic, SAE Technical Paper (2002) 2002-01-1962.

[12] S.S. Zhang, J. Power Sources 164 (2007) 351-364.

[13] V. Etacheri, R. Marom, R. Elazari, G. Salitra, D. Aurbach, Energy Environ. Sci. 4 (2011) 3243-3262.

[14] L.-X. Yuan, Z.-H. Wang, W.-X. Zhang, X.-L. Hu, J.-T. Chen, Y.-H. Huang, J.B. Goodenough, Energy Environ. Sci. 4 (2011) 269-284.

[15] A. Henry, Thermal transport in polymers, In: Annual Review of Heat Transfer, G. Chen, V. Prasad, Y. Jaluria (Eds.), Begell House, Danbury, CT, USA (2014).

[16] H. Maleki, S.A. Hallaj, J.R. Selman, R.B. Dinwiddie, H. Wang, J. Electrochem. Soc. 146 (1999) 947-954.

[17] J. Nanda, S.K. Martha, W.D. Porter, H. Wang, N.J. Dudney, M.D. Radin, D.J. Siegel, J. Power Sources 251 (2014) 8-13.

[18] J. Cho, M.D. Losego, H.G. Zhang, H. Kim, J. Zuo, I. Petrov, D.G. Cahill, P.V. Braun, Nature Communications 5 (2014) 1-6.

[19] V. Vishwakarma, A. Jain, J. Power Sources 272 (2014) 378-385. 
[20] P. Taheri, M. Bahrami, SAE Int. J. Passenger Cars 5 (2012) 164-176.

[21] U.S. Kim, C.B. Shin, C. Kim, J. Power Sources 189 (2009) 841-846.

[22] P.J. O’Brien, S. Shenogin, J. Liu, P.K. Chow, D. Laurencin, P.H. Mutin, M. Yamaguchi, P. Keblinski, G. Ramanath, Nature Mater. 12 (2012) 118-122.

[23] S. Kaur, N. Raravikar, B.A. Helms, R. Prasher, D.F. Ogletree, Nature Communications 5 (2014) 3082:1-8.

[24] J.P. Reifenberg, K.-W. Chang, M.A. Panzer, S. Kim, J.A. Rowlette, M. Asheghi, H.-S.P. Wong, K.E. Goodson, IEEE Electron Dev. Lett. 31 (2010) 56-58.

[25] J. Cho., Z. Li, E. Bozorg-Gayeli, T. Kodama, D. Francis, F. Ejeckam, F. Faili, M. Asheghi, K.E. Goodson, K.E., IEEE Trans. Components, Packag. Manufac. Technol. 3 (2013) 79-84.

[26] A. Javed, S. Iqbal, A. Jain, Appl. Phys. Lett. 101 (2012) 093707.

[27] A. Kasry, P. Borri, P.R. Davies, A. Harwood, N. Thomas, S. Lofas, T.Dale, ACS Appl. Mater. Interfaces 1 (2009) 1793-1798.

[28] V. Vishwakarma, C. Waghela, A. Jain, Microelectron. Eng. 142 (2015) 36-39.

[29] R. Prasher, Phys. Rev. B 77 (2008) 075424.

[30] J.R. Kim, S.W. Choi, S.M. Jo, W.S. Lee, B.C. Kim, J. Electrochem. Soc. 152 (2005) A295.

[31] R. Prasher, Appl. Phys. Lett. 94 (2009) 041905.

[32] K.L. Johnson, K. Kendall, A.D. Roberts, Proc. Roy. Soc., London, 324 (1971) 301-313.

[33] J.M. Baney, C.-Y. Hui, Adhesion Sci. Technol. 11 (1997) 393-406.

[34] K. Autumn, M. Sitti, Y.A. Liang, A.M. Peattie, W.R. Hansen, S. Sponberg, T.W. Kenny, R. Fearing, J.N. Israelachvili, R.J. Full, Proc. Nat. Acad. Sci., USA 99 (2002) 12252-12256.

[35] A. Alofi, G. P. Srivastava, Phys. Rev. B 87 (2013) 115421.

[36] E.T. Swartz, R.O. Pohl, Rev. Modern Phys., 61 (1989) 605. 
[37] B. Koo, P. Goli, A.V. Sumant, P.C. dos Santos Claro, T. Rajh, C.S. Johnson, A.A. Balandin, E.V. Shevchenko, ACS Nano 8 (2014) 7202-7207.

[38] F.D. Egitto, L.J. Matienzo, IBM J. Res. Development 38 (1994) 423-439.

[39] M.J. Shenton, M.C. Lovell-Hoare, G.C. Stevens, J. Phys. D: Appl. Phys. 34 (2001) 27542760 .

[40] http://ocw.mit.edu/courses/materials-science-and-engineering/3-11-mechanics-of-materialsfall-1999/modules/props.pdf, accessed March 17, 2015. 


\section{List of Figures:}

Figure 1. Thermal transport in the spiral geometry of a Li-ion cell: (A) Image of the spiral geometry of the electrode-separator roll extracted from a prismatic Li-ion cell; (B) Basic thermal unit cell that repeats itself in the direction normal to the electrode surface, and comprises of various material and interfacial thermal resistances; (C) Thermal resistances in the cathode-side half-cell considered in this work.

Figure 2. Schematics of three steady state temperature gradient based experiments. (A) separatorelectrode stack (Experiment 1); (B) single separator (Experiment 2), and (C) single cathode foil (Experiment 3) was placed between two copper blocks to determine various material and interface thermal resistances through measurement of total thermal resistance.

(MINOR REVISION) Figure 3. Measured temperature data from various thermocouples embedded in the copper blocks for Experiment 1. Extrapolated temperature difference determines temperature drop across the sample under test, and slope of the plot determines the heat flux. This results in measurement of total thermal resistance.

REVISED Figure 4. Summary of material-level thermal resistances in thermal unit cell of a Liion cell in baseline (Experiment A) and enhanced experiments (Experiment D). Colorbars show relative magnitudes of various resistances. Note that resistances due to the separator-cathode interface and separator must be counted twice in determining the total thermal resistance of the unit cell. $k_{\text {eff }}$ refers to the effective thermal conductivity of the unit cell, including interface thermal resistances. The materials and surface modifications for Experiments A and D are discussed in Figure 5.

REVISED Figure 5. Schematic showing the baseline experiment and various surface chemistry modifications explored for understanding the effect on thermal contact resistance between cathode and separator.

REVISED Figure 6. Comparison of the steady-state temperature response of baseline and three surface-modified separator-cathode stacks showing reduction in $R_{\text {total }}$. Note that the same heat flux is applied in each case. Once thermal contact resistance to copper blocks is subtracted out, the reduction in $R_{\text {sep-cathode }}$ due to surface treatment is even more significant.

REVISED Figure 7. Electrochemical evaluation of the baseline and thermally enhanced cathodes in a half-coin cell format with a Lithium counter electrode: (A) charge-discharge performance at $1.5 \mathrm{~mA}$, and (B) Electrochemical Impedance Spectroscopy (EIS) spectra taken at fully discharge state. A split flat cell with active area of $2.54 \mathrm{~cm}^{2}$ was assembled using Lithium anodes.

Figure 8. Cell-level thermal conductivity (left, blue) and peak temperature rise (right, green) as functions of $\mathrm{R}_{\text {sep-cathode, }}$ showing values corresponding to both baseline (circles) and enhanced (squares) thermal contact resistance. Measured values of the thermal contact resistance are shown on the x-axis. $k_{\text {eff }}$ data are based on calculation of the effective thermal conductivity of the 
thermal unit-cell, including thermal contact resistance. Temperature rise data are based on a recent analytical thermal model that predicts steady-state temperature distribution in a Li-ion cell. 
List of Tables:

Table 1. Thermal and mechanical properties for various materials for theoretical calculation of thermal contact resistance Note that since the calculation of thermal contact resistance was done from the Polyethylene side, only the heat capacity of Polyethylene is needed. 


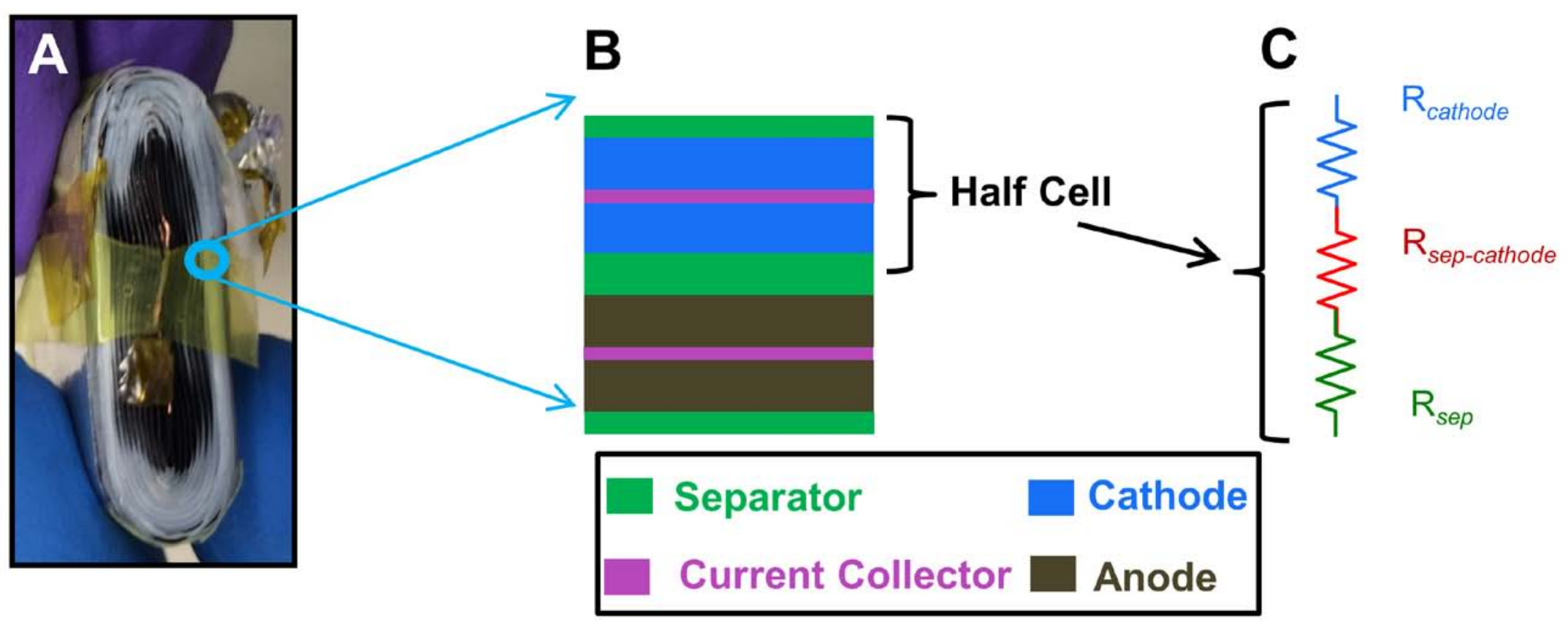

Figure 1. Thermal transport in the spiral geometry of a Li-ion cell: (A) Image of the spiral geometry of the electrode-separator roll extracted from a prismatic Li-ion cell; (B) Basic thermal unit cell that repeats itself in the direction normal to the electrode surface, and comprises of various material and interfacial thermal resistances; (C) Thermal resistances in the cathode-side half-cell considered in this work. 

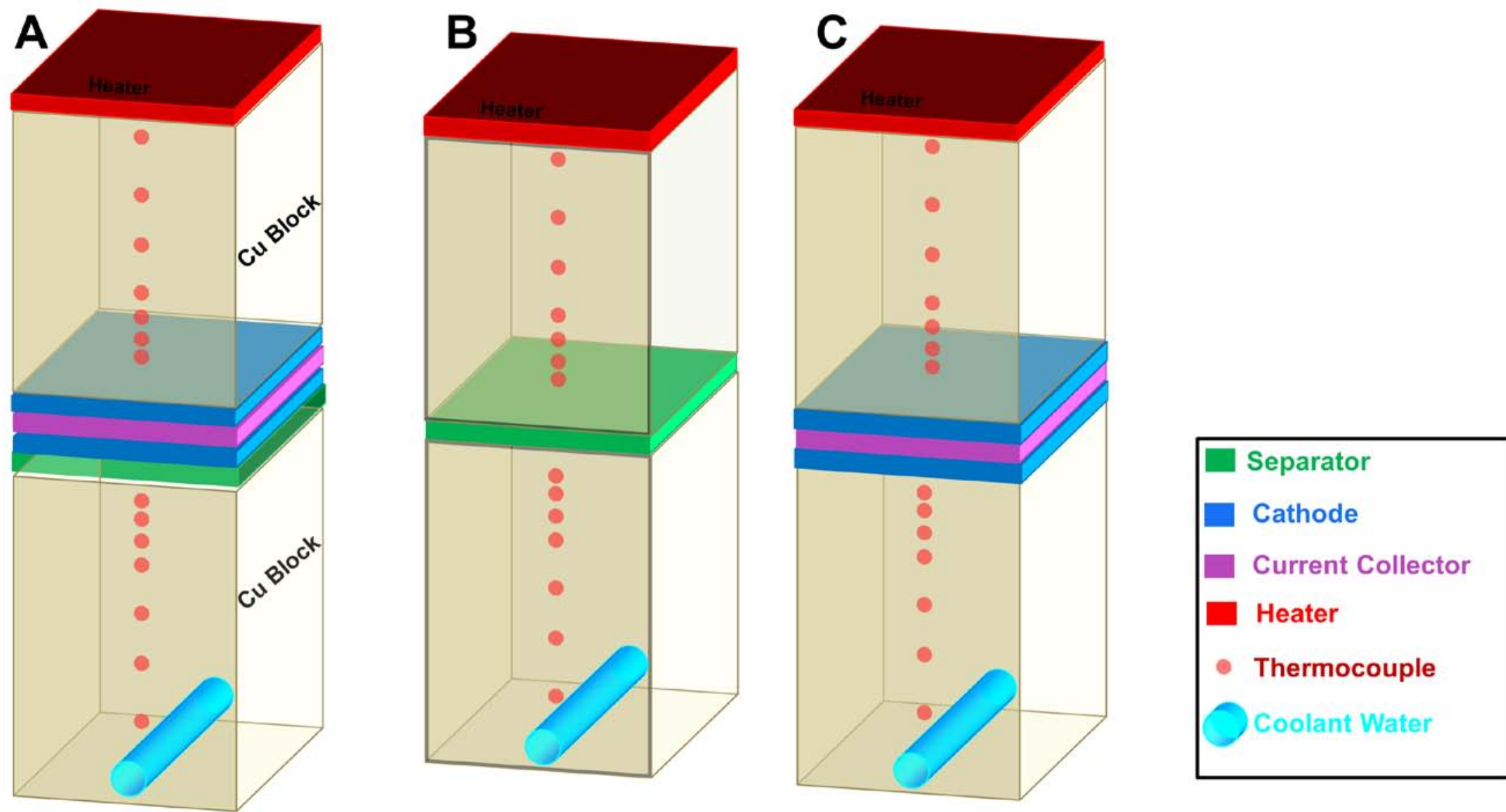

Figure 2. Schematics of three steady state temperature gradient based experiments. A (a) separator-electrode stack (Experiment 1), (b) single separator (Experiment 2), and (c) single cathode foil (Experiment 3) was placed between two copper blocks to determine various material and interface thermal resistances through measurement of total thermal resistance. 


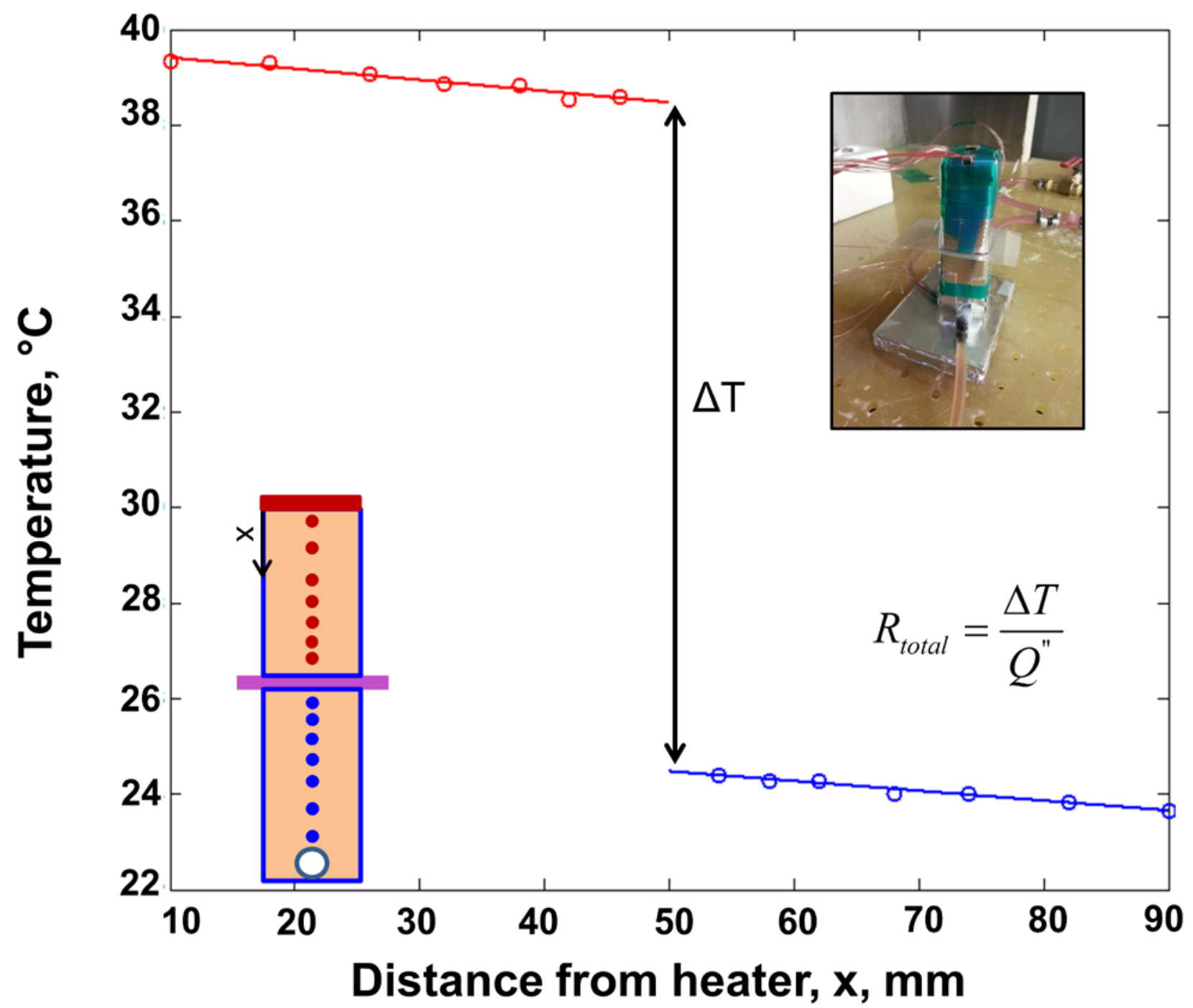

(MINOR REVISION) Figure 3. Measured temperature data from various thermocouples embedded in the copper blocks for Experiment 1. Extrapolated temperature difference determines temperature drop across the sample under test, and slope of the plot determines the heat flux. This results in measurement of total thermal resistance. 


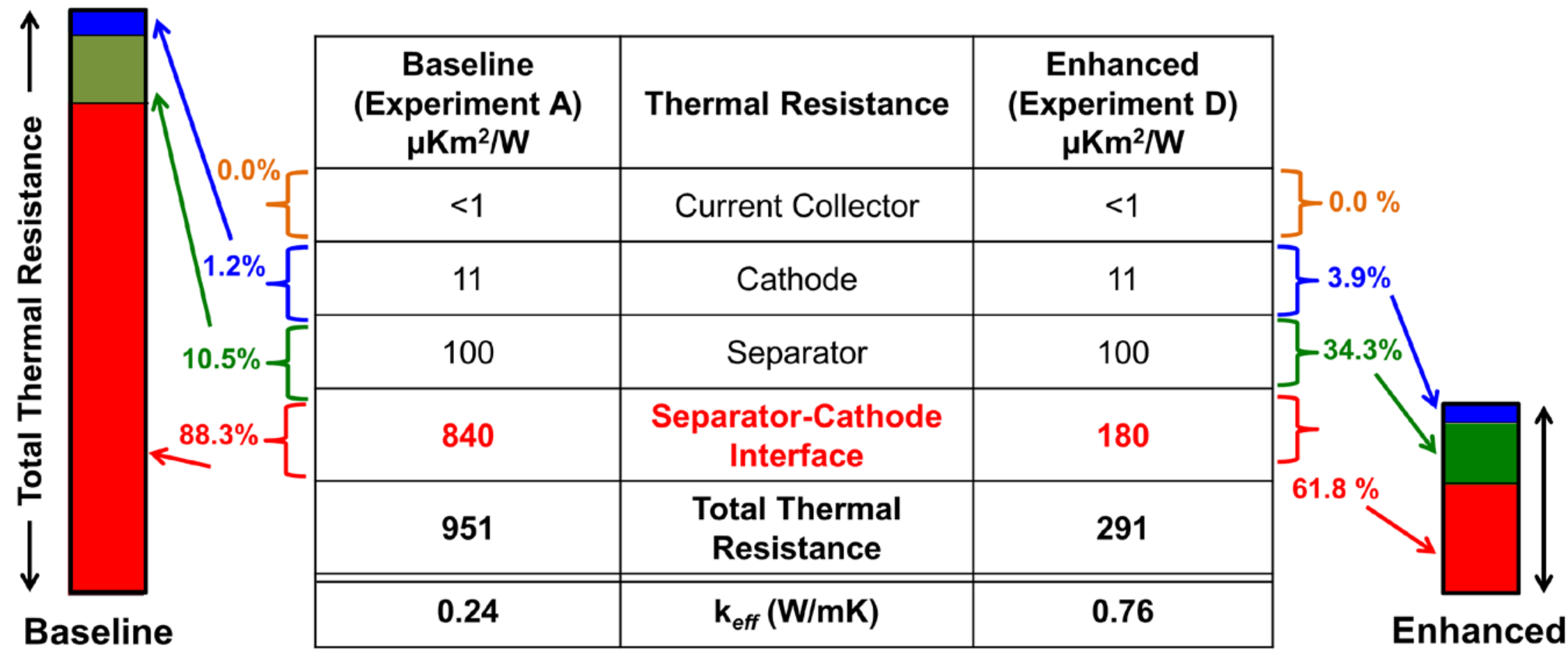

REVISED Figure 4. Summary of material-level thermal resistances in thermal unit cell of a Li-ion cell in baseline (Experiment A) and enhanced experiments (Experiment D). Colorbars show relative magnitudes of various resistances. Note that resistances due to the separator-cathode interface and separator must be counted twice in determining the total thermal resistance of the unit cell. $k_{e f f}$ refers to the effective thermal conductivity of the unit cell, including interface thermal resistances. The materials and surface modifications for Experiments A and D are discussed in Figure 5. 
Experiment A: Baseline experiment

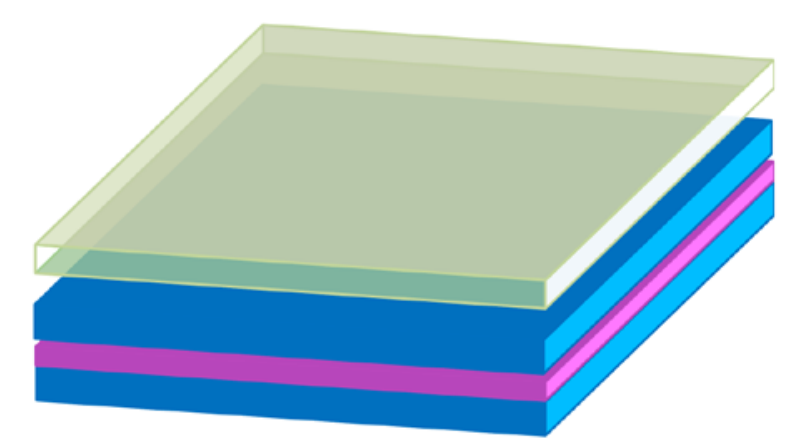

Experiment B:

Amine Functionalization of Cathode

\section{Separator}

Current Collector

\section{Cathode}

(1)

\section{Experiment C:}

Plasma Treatment

of Separator

\section{Experiment D:}

Both Plasma

Treatment \& Amine

Functionalization

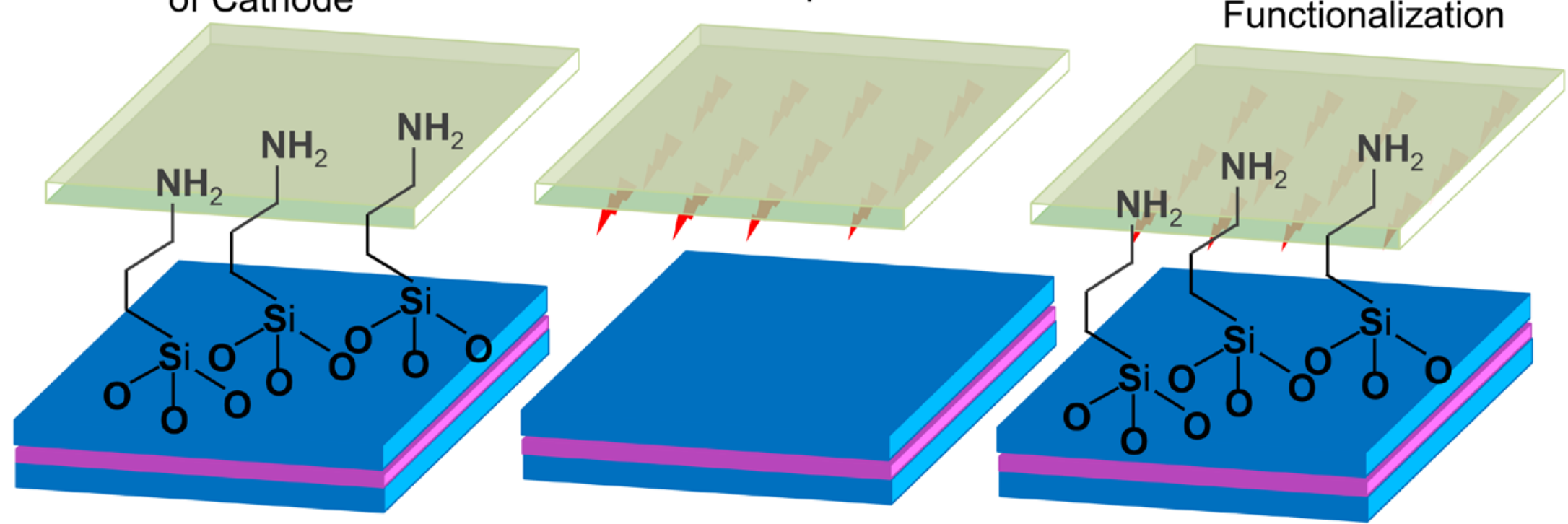

REVISED Figure 5. Schematic showing the baseline experiment and various surface chemistry modifications explored for understanding the effect on thermal contact resistance between cathode and separator. 


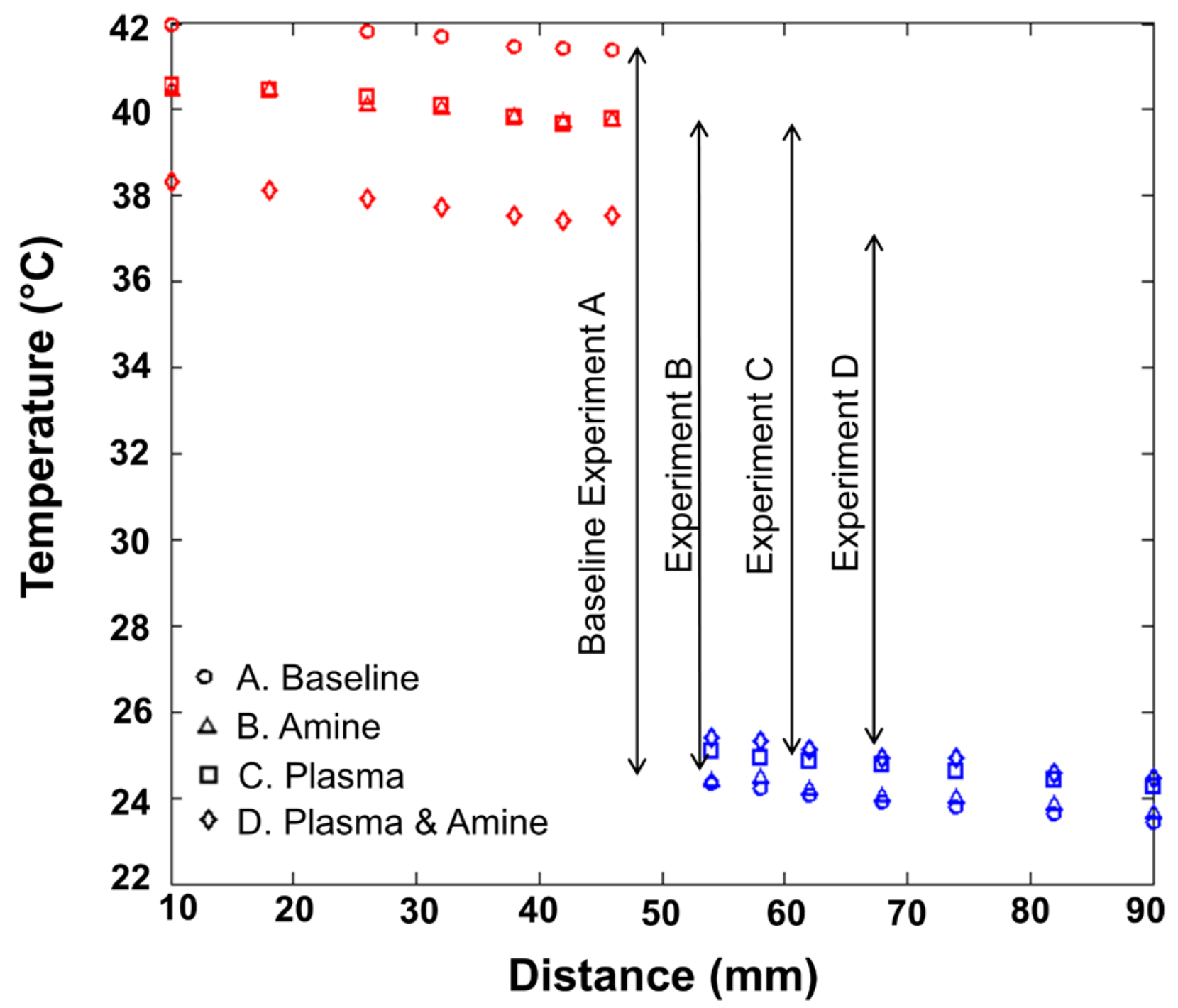

REVISED Figure 6. Comparison of the steady-state temperature response of baseline and three surface-modified separator-cathode stacks showing reduction in $R_{\text {total }}$. Note that the same heat flux is applied in each case. Once thermal contact resistance to copper blocks is subtracted out, the reduction in $R_{\text {sep-cathode }}$ due to surface treatment is even more significant. 
A

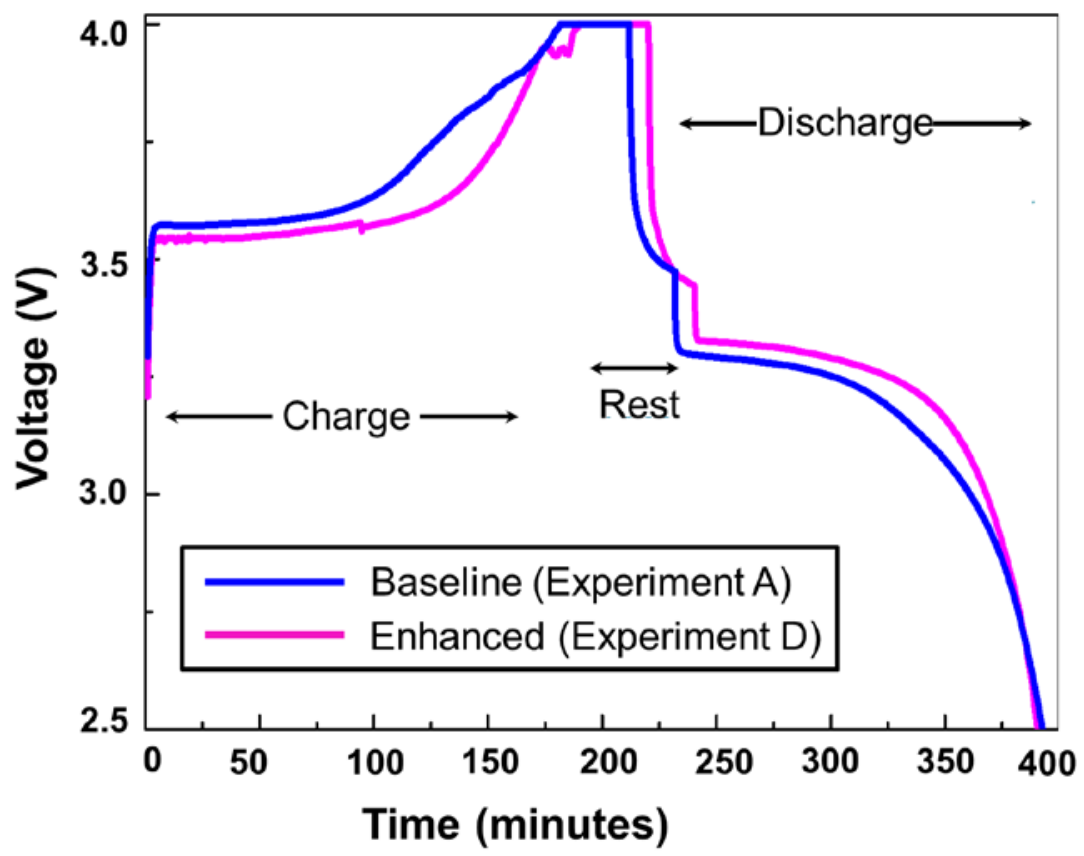

B

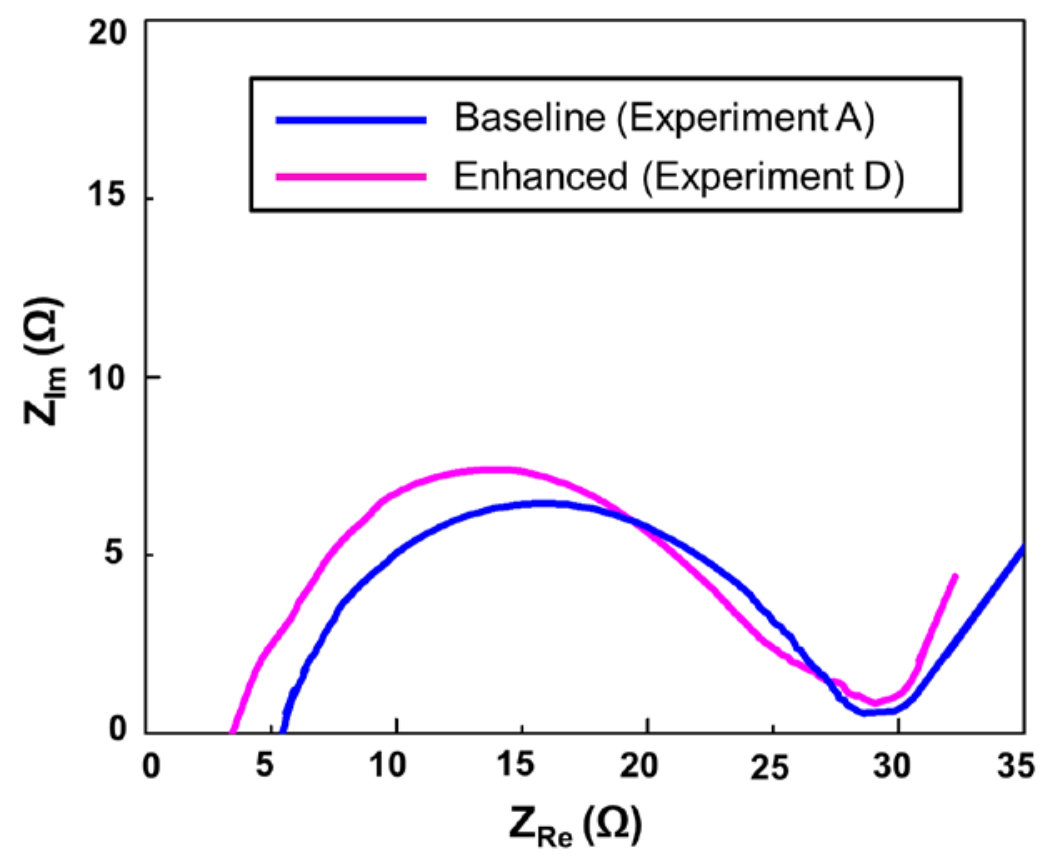

REVISED Figure 7. Electrochemical evaluation of the baseline and thermally enhanced cathodes in a half-coin cell format with a Lithium counter electrode: (A) charge-discharge performance at $1.5 \mathrm{~mA}$, and (B) Electrochemical Impedance Spectroscopy (EIS) spectra taken at fully discharge state. A split flat cell with active area of $2.54 \mathrm{~cm}^{2}$ was assembled using Lithium anodes. 


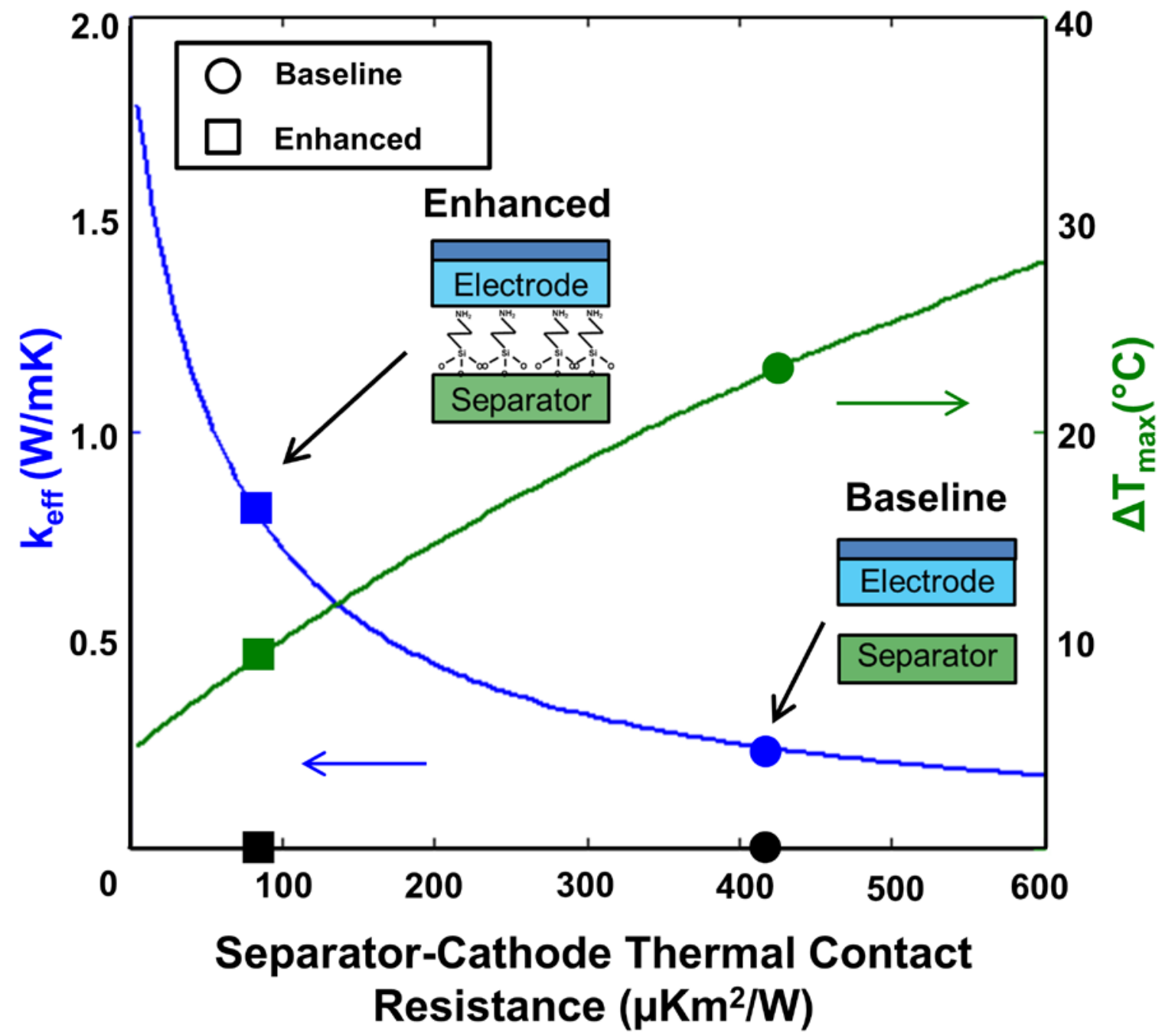

Figure 8. Cell-level thermal conductivity (left, blue) and peak temperature rise (right, green) as functions of $\mathrm{R}_{\text {sep-cathode, showing values }}$ corresponding to both baseline (circles) and enhanced (squares) thermal contact resistance. Measured values of the thermal contact resistance are shown on the x-axis. $k_{\text {eff }}$ data are based on calculation of the effective thermal conductivity of the thermal unit-cell, including thermal contact resistance. Temperature rise data are based on a recent analytical thermal model that predicts steady-state temperature distribution in a Li-ion cell. 
Table 1. Thermal and mechanical properties for various materials for theoretical calculation of thermal contact resistance Note that since the calculation of thermal contact resistance was done from the Polyethylene side, only the heat capacity of Polyethylene is needed.

\begin{tabular}{|c|c|c|c|c|c|}
\hline Material & $\begin{array}{c}\text { Effective speed } \\
\text { of sound }(\mathrm{m} / \mathrm{s})\end{array}$ & $\begin{array}{c}\text { Mass density } \\
\left(\mathrm{g} / \mathrm{cm}^{3}\right)\end{array}$ & $\begin{array}{c}\text { Heat capacity } \\
\left(\mathrm{J} / \mathrm{m}^{3}{ }^{\circ} \mathrm{C}\right)\end{array}$ & $\begin{array}{c}\text { Young's } \\
\text { modulus }(\mathrm{GPa})\end{array}$ & $\begin{array}{c}\text { Poisson's } \\
\text { ratio }\end{array}$ \\
\hline $\mathrm{LiCoO}_{2}[18]$ & 5020 & 5.06 & & 174 & 0.38 \\
\hline Polyethylene [40] & 672 & 0.95 & 1824000 & 0.7 & 0.42 \\
\hline Graphite [35] & 1940 & 2.26 & & 33 & 0.33 \\
\hline
\end{tabular}

